\title{
Introduction to Systems Engineering
}

\author{
Vincenzo Arrichiello \\ INCOSE Chapter Italia \\ vincenzo.arrichiello@incose.org \\ Copyright $\odot$ held by the authors.
}

\begin{abstract}
The word "system" is used in many areas (e.g. solar system, telephone system, nervous system, transportation system, ecological system, etc.) mainly to convey a meaning of ordering and/or interaction. The systems of interest for Systems Engineering are a specific subset: Engineered Systems. These are defined as "an integrated set of elements, subsystems, or assemblies that accomplish a defined objective." Having delimitated the area of application, what appears to be worth to be analyzed is if, and how, Systems Engineering differentiates itself from "Traditional Engineering".
\end{abstract}

A useful, and very concise, reference for the description of engineering (design) is the one from the Royal Academy of Engineering (Royal Academy of Engineering, 1999):

- Need - All design begins with a clearly defined need;

- Vision - All designs arise from a creative response to a need;

- Delivery - All designs result in a system, product or project which meets the need.

That description applies, without any doubt, also to Systems Engineering, so one is left wondering whence the need for it originated. Looking more in detail, one can observe that "Traditional" Engineering activities have been typically limited to a definite discipline, with practitioners very likely to become "experts" in the relevant field. The design approach usually applied in these conditions is the "Solution-oriented" approach, "in which an initial solution is proposed, analyzed and then repeatedly modified as the design space and requirements are explored together" (Wynn and Clarkson, 2005)." This approach is effective when the designer, thanks to her/his experience, is able to devise an initial solution that is not too far from the "ideal" final one. On the contrary, when the solution to the problem at hand spans multiple engineering disciplines (thus making very unlikely that a single person can master all of them) this approach proves far less effective, if not a true recipe for disaster.

Likely due to the complexity that increasingly tends to be part also of "traditional" engineering endeavors, the design process has largely evolved to the "Problem-oriented" approach ," in which the emphasis is placed upon abstraction and thorough analysis of the problem structure before generating a range of possible solutions." (Wynn and Clarkson, 2005) This approach bears a strong resemblance to the Systems Engineering process, and it is somewhat telling that it is defined a "Systematic Design Process".

Systems Engineering thus appears to have somewhat anticipated this trend of evolution; a survey of the context in which it first appeared may be of help to better understand its peculiar characteristics. The term "system" referred to engineering activities and products, makes its first appearance in organizations dealing with electric power distribution and telephone networks (notably, AT\&T and Bell Labs), in the first half of the twentieth century. Systems Engineering, in the form of the organized interdisciplinary approach as it is known today, appears in the early fifties of that century. Its emergence can be mainly tracked to the concurrence of novelties appearing in three contexts: cultural, technological and historic. The 
three elements of the cultural context of major relevance to Systems Engineering are: General Systems Theory, Cybernetics and Operations Research.

Heralding the birth of the systems science, in 1950 Ludwig von Bertalanffy outlined the "General System Theory" (GST) (Bertalanffy, 1968). He was driven by the observation of the limits shown by classical scientific methods when dealing with entities (both biological and technological) consisting of parts characterized by strong, nonlinear interactions. Noting that such entities cannot be understood by investigating their respective part in isolation, Bertalanffy introduces the notion of wholeness: the need to think "in terms of systems of elements in mutual interaction." Knowing not only the parts, but also the relations, enables to understand the characteristics of the whole which depend on the relations between its components, and are not explainable from the sole characteristics of isolated parts; those characteristics therefore appear as "new" or "emergent."

Another GST tenet is the concept of Open system (contrasted with the "closed system" typical of reductionism). An open system cannot be isolated by its environment; on the contrary this last must be thoroughly taken into account in order to understand the system and its behavior. Bertalanffy also highlights as fundamental the concept of hierarchic order, which was further developed by other scholars.In the same years, science was developing new understandings in the fields of information and automatic control, mainly thanks to the works of Claude Shannon, Warren Weaver and Norman Wiener.

Applying a broad outlook spanning from technology to biology up to social systems, Wiener coined the term "Cybernetics" to denote a new field of science devoted to the scientific study of "Control and Communication in the Animal and the Machine (Wiener, 1948)." Feedback (defined as "the property of being able to adjust future conduct by past performance") (Wiener, 1950) is the central concept of Cybernetics. Wiener classes communication and control together, and portrays feedback as a two-way exchange of information between the system and its environment. This way he somewhat extends Bertalanffy's open systems concept by seeing the "automata" coupled to the external world, not merely by a flow of energy, but also by a flow of incoming and outgoing messages.

In a previous work (Rosenblueth, Wiener and Bigelow, 1943) Wiener had defined the "behavioristic approach", consisting in the "examination of the output of the object and of the relations of this output to the input" equally "applicable to both machines and living organisms." This, on one side allows to define the term "black box" to indicate an element performing a definite operation "but for which we do not necessarily have any information of the structure by which this operation is performed" (Wiener, 1948), as contrasted with a "white box" in which internal structure is determined and known. On the other side the approach of treating in the same way machines and living organisms, favored the trend to see systems as a combination of interconnected elements coordinating together toward a specific purpose (the "organic analogy"; e.g. depicting an Air Defense System as an "organism" possessing "sensory components, communication facilities, data analyzing devices, centers of judgment, directors of action, and effectors, or executing agencies") (PRADSEC, 1950).

Operations Research originated during World War II as a means to support decisions about the most effective employment of resources; it proved highly successful and, after the war, it was applied also in fields other than the military. The same methods of mathematical modelling and statistical analysis lend themselves well also to evaluate the expected effectiveness of alternative potential (not yet existing) solutions, hence the approach (now re-named "Systems Analysis") was soon developed; it is also applied to the analysis of the specifications of new systems. The Operations Research groups included, in addition to mathematicians, physicists and engineers, a variety of representatives of other sciences; thus it 
had the additional effect to demonstrate the effectiveness of the "mixed team approach"; as Weaver (Weaver, 1948) notes "it was found that members of such diverse groups could work together and could form a unit which was much greater than the mere sum of its parts."

While Systems Engineering originated somewhat independently, the novel concepts of systems science had likely some influence on its development and provided an underlying foundation for its progressive conceptualization.

From the point of view of the technological context, the same years are characterized by the development of a profusion of novel technologies, either totally new or in fast evolution (e.g. radar, communications, automatic control, inertial navigation, computing, rocketry, etc.). The potential new developments that could be made possible by applying and combining the new technologies proved an enticement hard to resist both for users and technologist, although it was easy to foresee that difficulties would likely be encountered.

The historical context was marked by the beginning of the cold war and of the related arm race and space race. This involved the launch of unprecedented, large-scale and high complexity projects, which combined challenging goals with time pressure. It must be noted that the same context provided for a strong motivation, as the involved people felt to be working for a worthy cause.

The quintessential projects marking the origins of Systems Engineering are the Intercontinental Ballistic Missile (ICBM) and the Semi-Automatic Ground Environment (SAGE) projects. The ICBM project faced the daunting task to integrate a variety of novel and heterogeneous technologies (many still under development) to attain challenging performances under severe constrains in terms of dimensions, weight and severity of the environment. This not only frequently gave rise to unanticipated adverse consequences, but also required that experts of diverse discipline worked effectively in large multidisciplinary teams. Progresses were hampered not only by the substantial technical problems, but also by more mundane aspects, like: poor communication, lack of planning and coordination, inconsistent development of components and poor control of the configuration.

Tackling these problems spurred the creative development of management processes; these represent the first instantiation of the systems engineering methodology (e.g. Systems Management, Configuration Management, Extensive Testing, Data management and communications). Further application of these methods for subsequent projects (culminating with the Apollo program) led to their refinement and eventual formalization into the SE process. The SAGE project, on its side, mainly anticipated two aspects that were posed to become of major relevance for modern Systems Engineering: Software development and Human System Integration.

The first required the development of procedures and approaches for the ordered development of "computer programs"; the second required both the understanding of the operational processes to be supported by the system, and the development of human-machine interfaces enabling an effective integration of the operators with the system. These methodologies eventually evolved in disciplines (Software Engineering and Human System Integration) which, although separate, are closely related to Systems Engineering.

The effects of these cultural, technological and historical factors likely concurred in determining the duality of Systems Engineering; that is, its combination of two complementary aspects:

- Systemic - refers to the holistic appreciation of the problem/system of interest, considering its context, stakeholders, and the interrelationships and interconnections; 
- Systematic - refers to taking a structured, orderly approach to solve the problem and to implement the system.

- The Systemic Approach (also referred to as "Systems Approach" and "Systems Thinking") is mostly a way of thinking; its foundations can be clearly tracked to the concepts and principles of the system science:

- Wholeness (focus on the parts AND their interactions);

- Emergence (characteristics deriving from the interactions between the parts);

- Hierarchy (to contain and conceal complexity);

- Behavior (focus on the relation between output and input, independent of the structure and the internal organization - "Black Box" model);

- Environment (the system affects and is affected by the environment);

- Prediction of performances (through Modelling and Simulation).

This is confirmed by the definition from the SeBoK (SeBoK, 2014): "the systems approach to engineered systems is a problem-solving paradigm. It is a comprehensive problem identification and resolution approach based upon the principles, concepts, and tools of systems thinking and systems science, along with the concepts inherent in engineering problem-solving. It incorporates a holistic systems view that covers the larger context of the system, including engineering and operational environments, stakeholders, and the entire life cycle."

On its part, the prescriptive methodology that forms the reference for the systematic approach has its all too apparent roots in the processes and management solutions firstly developed for the trailblazing projects. A sequence of standards, from the "375 Series" (Air Force Systems Command) to the ISO/IEC 15288 (ISO/IEC 15288, 2008) documents its evolution to the present days.

The process that is captured by the more recent standards is well proved and effective, providing a valuable reference for the practitioner. What must be kept in mind is that a process can record only the explicit knowledge; a primary role in the discovery and understanding of needs, the articulation of functions and behaviors, and the devising of suitable architectures is played by knowledge and skills (intuition, ingenuity, critical thinking) which is very hard to codify. What can be done is trying to define some "principles" that can provide some guidance (although not being prescriptive rules suitable for unthinking adherence).

One principle that can help in understanding how Systems Engineering can prove (and indeed has proven) highly effective in dealing with complex multidisciplinary engineering problems is the so called "left shift." The term refers to a specific shape of the resource profile of a project, which exhibits an earlier peak (positioned more on the left in a diagram with the horizontal time axis directed to the right, hence the name). This early commitment of resources can potentially prove beneficial in two main ways. On one side, it can help in anticipating the discovery of errors and omissions before they manifest themselves in a later stage of development; it is well known that the cost of fixing problems increases significantly along a project lifecycle, thus an earlier fixing can reduce the cost of the project (and often saving time, too). On the other side, a large part of the total cost of a project is committed in the early phases of its lifecycle (about 70\% in the concept phase) (Haskins, 2011); a "frontloading" of resources in the early phases can enable to proactively build a suitable problem-specific knowledge basis, and to perform analyses, both of which are needed to enable well-grounded design decisions. If this is missing, knowledge will be acquired through a trial and error process, for sure not in a timely way to avoid adverse consequences. It must be evidenced that, due to the probabilistic nature of the pay-off (costs avoided), this approach must be seen as an investment (Emes, Smith, James, Whyndham, Leal and Jackson, 2012). 
An interesting, and inspiring, collection of "essential ingredients of Systems Engineering" is presented by Boardman and Sauser (Boardman and Sauser, 2008); one could even semi-seriously label them the "Seven Pillars" of Systems Engineering:

- Life cycle - to carefully consider the entire life-cycle of the system, from conception to retirement, primarily in the temporal dimension, but also accounting for the evolutions in the contextual and stakeholder dimensions occurring as the life-cycle unfolds.

- Gates - are intended to ensure a safe progression along the project lifecycle; they are associated with reviews and baselines. Baselines must be defined so they can provide rock-solid foundations for further work. Reviews offer the opportunity to share views and to make sense of progress from all angles.

- Requirements - are a way to connect and at the same time keep separate the problem space and the solution space, translating needs into requirements while preserving a design space as wide as possible. Requirements elicitation and derivation entails a thorough exploration and understanding of both the problem and the solution spaces.

- Perspectives - the diverse stakeholders (persons with a legitimate interest in the system) must be discovered alongside the viewpoints that each of them has (at least one each).

- Trade-off - design is a matter of decisions (what to do, how to do, who does what); in any decision, candidates must be identified, criteria formulated, performances evaluated and a selection made. The duty is to facilitate rational thinking and to report as rationally as possible when irrational decisions are made.

- Modelling and Simulation - Models allow for prediction of effectiveness and performances, performing experiments without the "real thing". Since they are abstraction of reality, they are "all wrong", but useful if properly built for a specific purpose.

- Operational effectiveness - to provide a high-quality product that will serve the interests of the customer in terms of both technical and business needs requires a long-term vision for a project right at its beginnings. In addition to performance, availability must be assured and complemented by process effectiveness (taking into account that the system is embedded in a wider system); one last, but not less important, element in defining Operational Effectiveness is Total Ownership Costs.

\section{References}

Royal Academy of Engineering. 1999. Principles of Engineering Design Wynn D. and Clarkson J., 2005. Models of designing, in Design Process Improvement: A review of current practice, J.Clarkson and C.Eckert

Bertalanffy, L. von, 1968. An outline of general systems theory, British Journal for the Philosophy of Science 01/1950 and General System Theory: Foundations, Development, Applications, Braziller

Wiener N., MIT Press, 1948. Cybernetics: Or Control and Communication in the Animal and the Machine

Wiener N., 1950. The Human Use of Human Beings

Rosenblueth A., Wiener N. and Bigelow J., 1943. Behavior, Purpose and Teleology, Philosophy of Science

PRADSEC, 1950. Progress Report of the Air Defense System Engineering Committee Weaver W., 1948. Science and complexity, American Scientist

SeBoK, 2014. Retrieved on http://www.sebokwiki.org/wiki/Systems_Approach Applied_to_Engineered_Systems 
U.S. Air Force Systems Command, AFSCM 375-5 SYSTEM ENGINEERING MANAGEMENT PROCEDURES, 196

ISO/IEC 15288-2008(E), 2008. Systems and software engineering - System life cycle processes

Haskins C. ed. 2011, "INCOSE Systems Engineering Handbook v. 3.2.2

Emes, MR; Smith, A; James, AM; Whyndham, MW; Leal, R; Jackson, SC, 2012. Principles of systems engineering management: Reflections from 45 years of spacecraft technology research and development at the mullard space science laboratory. Proc. of the 22nd Annual International Symposium of the International Council on Systems Engineering (INCOSE)

Boardman J., Sauser B., 2008. Systems thinking : coping with 21st century problems, Taylor \& Francis 


\section{Biography}

Vincenzo Arrichiello holds a Laurea Degree in Engineering from the University of Genoa. He has practiced Systems Engineering for about 30 years, covering positions either in large companies (Defense Contractors) and engineering consulting Companies (as a co-owner), applying the systems approach across diverse industry fields (Aerospace\& Defense, Robotics, Packaging) He was one of the founders of the Italia Chapter of INCOSE and is now serving as its President. From 2009 he is head of the Selex Academy, and directs the relevant Systems Engineering School. He teaches Systems Engineering courses in the Academy and in some University Masters. 